\title{
CD55 and CD59 expression protects HER2-overexpressing breast cancer cells from trastuzumab-induced complement-dependent cytotoxicity
}

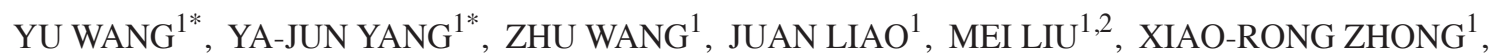 \\ $\mathrm{HONG} \mathrm{ZHENG}^{1}$ and YAN-PING WANG ${ }^{1}$ \\ ${ }^{1}$ Laboratory of Molecular Diagnosis of Cancer, West China Hospital, Sichuan University, Chengdu, Sichuan 610041; \\ ${ }^{2}$ Breast Cancer Research Center, Chongqing Cancer Institute, Chongqing 400030, P.R. China
}

Received October 8, 2015; Accepted April 28, 2017

DOI: $10.3892 / \mathrm{ol} .2017 .6555$

\begin{abstract}
A large proportion (40-60\%) of patients with human epidermal growth factor receptor 2 (HER2)-overexpressing breast cancer do not benefit from trastuzumab treatment, potentially due to the lack of complement-dependent cytotoxicity (CDC) activation. In the present study, the effect of complement decay-accelerating factor (CD55) and CD59 glycoprotein precursor (CD59) expression on trastuzumab-induced CDC in HER2-positive breast cancer cell lines was investigated. The CD55 and CD59-overexpressing and HER2-positive cell lines SK-BR-3 and BT474 were selected for subsequent experiments. Blocking CD55 and CD59 function using targeting monoclonal antibodies significantly enhanced the cell lysis of SK-BR-3 and BT474 cells following treatment with trastuzumab. In addition, following treatment with $0.1 \mathrm{U} / \mathrm{ml}$ phosphatidylinositol-specific phospholipase C (PI-PLC) for $1 \mathrm{~h}$, CD55 and CD59 surface expression was significantly decreased, and the cell lysis rate was further enhanced. Treatment of SK-BR-3 cells with short hairpin RNA (shRNA) targeting CD55 and CD59 downregulated CD55 and CD59
\end{abstract}

Correspondence to: Professor Yan-Ping Wang, Laboratory of Molecular Diagnosis of Cancer, West China Hospital, Sichuan University, 37 Guoxue Lane, Chengdu, Sichuan 610041, P.R. China E-mail: wyanping@scu.edu.cn

"Contributed equally

Abbreviations: CDC, complement-dependent cytotoxicity; PI-PLC, phosphatidylinositol-specific phospholipase C; ADCC, antibody-dependent cellular cytotoxicity; mAbs, monoclonal antibodies; mCRPs, membrane-bound complement regulatory proteins; shRNA, short hairpin RNA; NHS, normal human serum; INHS, inactivated normal human serum; FCM, flow cytometry; BSA, bovine serum albumin; MFI, mean fluorescence intensity

Key words: human epidermal growth factor receptor 2, trastuzumab, CDC, CD55, CD59, breast cancer expression at the mRNA and protein levels, and resulted in significantly enhanced trastuzumab-induced CDC-dependent lysis. The data from the present study suggested that CD55 and CD59 serve roles in blocking trastuzumab-induced CDC, therefore strategies targeting CD55 and CD59 may overcome breast cancer cell resistance to trastuzumab. The results from the present study may provide a basis for developing suitable, personalized treatment strategies to improve the clinical efficacy of trastuzumab for patients with HER2-positive breast cancer.

\section{Introduction}

Breast cancer is one of the most common malignant tumors in women worldwide (1). Receptor tyrosine-protein kinase erbB2/human epidermal growth factor receptor 2 (ERBB2/HER2) is a transmembrane tyrosine kinase receptor that belongs to the family of epidermal growth factor receptors. HER 2 is expressed in $25-30 \%$ of invasive breast cancers and is associated with invasion, metastasis and prognosis (2). Trastuzumab (product name Herceptin) is the first recombinant humanized monoclonal antibody directed against the extracellular domain of HER2 that has been approved by the US Food and Drug Administration for the treatment of HER2-positive breast cancer. However, 40-60\% of patients with HER2-positive breast cancer do not benefit from it (3). The molecular mechanisms underlying acquired resistance to trastuzumab remain poorly understood.

Trastuzumab has several possible mechanisms of action, which include specific binding to HER2 and blocking of ligand-mediated cell signaling, inhibition of cell growth, inducing apoptosis, inhibition of tumor angiogenesis, and improving the ability of immune cells to target tumor cells through antibody-dependent cellular cytotoxicity (ADCC). ADCC and complement-dependent cytotoxicity (CDC) are innate immune mechanisms that destroy tumor cells and serve important roles in mediating the effects of therapeutic monoclonal antibodies (mAbs) in the treatment of cancer (4). Examples of therapeutic mAbs include rituximab, alemtuzumab and cetuximab. However, it has been reported that CDC does not serve a role in mediating the effects of 
trastuzumab $(5,6)$. Since the contribution of the complement system to the anti-tumor effect of trastuzumab remains unclear, the deficiency of CDC may explain why the majority of patients with HER2-positive cancer are not sensitive to trastuzumab. The efficiency of CDC against tumor primarily depends on the activation of the complement system, which is regulated by membrane-bound complement regulatory proteins (mCRPs), including complement decay-accelerating factor (CD55) and CD59 glycoprotein precursor (CD59). A number of types of cancer have been reported to escape from the complement attack due to high expression of mCRPs, which protects cancer cells from CDC and anticancer immune responses $(7,8)$.

A previous study from our lab demonstrated that CD55 and CD59 were two important inhibitors of CDC triggered by heterologous expression of $\alpha$-gal xenoantigen in colon tumor cell lines (9). In addition, patients with breast cancer that overexpressed CD55 or CD59 exhibited a higher relapse rate following trastuzumab treatment compared with those with low expression of CD55 or CD59 (10). The mean disease-free survival of patients with CD55 or CD59 overexpression was significantly shorter compared with those with low expression of CD55 or CD59, while the expression of CD46 had no effect on prognosis.

It was hypothesized that CDC induced by trastuzumab in HER2-positive breast cancer may be limited due to overexpression of CD55 and CD59. The aim of the present study was to investigate the association between CD55/CD59 expression and trastuzumab-induced CDC in a HER2-positive breast cancer cell line. This was achieved by blocking CD55/CD59 activity using mAbs, silencing their expression using short hairpin RNA (shRNA), and modulating their expression via phosphatidylinositol-specific phospholipase C (PI-PLC).

\section{Materials and methods}

Cell lines. The human breast cancer BT474, SK-BR-3, ZR-75-1, MDA-MB-468, MCF-7, BT549 and MDA-MB-231 cell lines, and a human colorectal adenocarcinoma (LoVo) and pig iliac artery endothelial (PIEC) cell line were purchased from the Cell Bank of Type Culture Collection of the Chinese Academy of Sciences (Shanghai, China). PIEC, which naturally expresses high level of $\alpha$-gal xenoantigen, was used as a positive control to assess complement activity in cytolysis assays. MCF-7, BT549, LoVo and PIEC cells were cultured in RPMI-1640 medium containing 10\% fetal bovine serum from Gibco (Thermo Fisher Scientific, Inc., Waltham, MA, USA). SK-BR-3, MDA-MB-468, MDA-MB-231 and ZR-75-1 cells were cultured in DMEM medium containing $10 \%$ fetal bovine serum (Gibco; Thermo Fisher Scientific, Inc.) and 100 IU/ml penicillin/streptomycin (North China Pharmaceutical Co., Ltd., Shijiazhuang, China). BT474 cells were cultured in MEM medium containing 10\% fetal bovine serum (Gibco; Thermo Fisher Scientific, Inc.), $100 \mathrm{IU} / \mathrm{ml}$ penicillin/streptomycin (North China Pharmaceutical Co., Ltd.) and $40 \mathrm{U} / \mathrm{ml}$ insulin from Tonghua Dongbao Pharmaceutical Co., Ltd. (Jilin, China). The cells were maintained in an incubator at $37^{\circ} \mathrm{C}$ in a $5 \% \mathrm{CO}_{2}$ humidified atmosphere.

Sera. Pooled normal human serum (NHS) used as the source of complement and anti- $\alpha$-gal-specific antibodies was provided by the central blood bank at West China Hospital, Sichuan University (Chengdu, China) and stored at $-80^{\circ} \mathrm{C}$ in aliquots until assayed. Diluted (50\%) NHS was screened using a PIEC killing efficiency assay, as described previously (9), and only sera with $100 \%$ PIEC killing efficiency was utilized for subsequent experiments. Pooled heat-inactivated normal human serum (INHS) was obtained by incubating the sera at $56^{\circ} \mathrm{C}$ for $30 \mathrm{~min}$, which was used as negative control in all experiments.

Antibodies and reagents. Fluorescein isothiocyanate (FITC)-conjugated mouse anti-human CD55 (cat no. FHF055) and CD59 (cat no. FHF0591) mAbs and a FITC-conjugated immunoglobulin $\mathrm{G}(\mathrm{IgG}) 1$ isotype Control $\mathrm{mAb}$ (cat no. GMP01103F) for flow cytometry (FCM) were purchased from 4A Biotech Co., Ltd. (Beijing, China). Mouse anti-HER2/ c-erbB-2 monoclonal antibody (cat no. ZM-0065) and Biotin-Streptavidin HRP detection systems (cat no. SP-9000) for immunohistochemistry was purchased from Origene Technologies (Beijing, China). The blocking mAb against CD55 (cat no. BRIC216) was purchased from EMD Millipore (Billerica, MA, USA). The blocking mAb against CD59 (cat no. MFM-43) was purchased from Abcam (Cambridge, UK). The mAbs against CD55 (BRIC216), CD59 (YTH53.1) and $\beta$-actin (cat no. SC-47778) for western blot analysis were purchased from Santa Cruz Biotechnology, Inc. (Dallas, TX, USA). The horseradish peroxidase (HRP)-labeled goat anti-mouse IgG (H+L, cat no. 170-6516) was from Bio-Rad Laboratories, Inc. (Hercules, CA, USA). The HRP-labeled goat anti-rat IgG $(\mathrm{H}+\mathrm{L}$, cat no. sc-2006) was from Santa Cruz Biotechnology, Inc.

Trastuzumab was obtained from Genentech, Inc. (San Francisco, CA, USA). PI-PLC $100 \mathrm{U} / \mathrm{ml}$ and Lipofectamine ${ }^{\circledR} 2000$ were purchased from Invitrogen (Thermo Fisher Scientific, Inc.).

Plasmids and primers. The specified pGPU6/GFP/Neo-shRNA expression plasmids (shCD55 and shCD59), and the negative control pGPU6/GFP/Neo-shNC (shNC) were designed and produced by GenePharma Co., Ltd. (Shanghai, China). The shCD59 targeted sequence 5'-TGAGCTAACGTACTACTA CTGC-3' was designed according to Shi et al (11). The four specified shCD55 targeted sequences included shCD55/545, 5'-GCAGTCAATGGTCAGATATTG-3'; shCD55/613, 5'-GCA TCCCTCAAACAGCCTTAT-3'; shCD55/829, 5'-GGCATA TTATTTGGTGCAACC-3' and shCD55/1075, 5'-GGAGAG CACTCTATTTATTGT-3'. The shNC targeted sequence was 5'-GTTCTCCGAACGTGTCACGT-3'. The primers for reverse transcription-quantitative polymerase chain reaction (RT-qPCR) analysis of CD55, CD59 and GAPDH were synthesized by Sangon Biotech Co., Ltd. (Shanghai, China) as follows: CD55 forward, 5'-TTCCCCCAGATGTACCTAATGC-3' and reverse, 5'-TTACAGTATCCTCGGGAAAACTTGT-3'; CD59 forward, 5'-TAACCCAACTGCTGACTGCAA-3' and reverse, 5'-TTTGGTAATGAGACACGCATCAA-3'; GAPDH forward, 5'-GAAGGTGAAGGTCGGAGTC-3' and reverse, 5'-GAAGATGGTGATGGGATTTC-3'.

Detection of CD55 and CD59 expression by flow cytometry. Cells were removed from the culture flask using $0.25 \%$ trypsin 
and $0.25 \%$ EDTA, washed with $1 \%$ bovine serum albumin (BSA) diluted in PBS and centrifuged at $300 \mathrm{x}$ g for $10 \mathrm{~min}$, then suspended in $100 \mu \mathrm{l} 1 \% \mathrm{BSA}$ and incubated with $10 \mu \mathrm{l}$ FITC-CD55 or FITC-CD59mAbs for $30 \mathrm{~min}$ at $37^{\circ} \mathrm{C}$. Flow cytometry was performed using a FACSAria I and data were analyzed using FACSDiva 6.0 (both from BD Biosciences, Franklin Lakes, NJ, USA). Cells used FITC-IgG1 isotype control $\mathrm{mAb}$ as the negative control. To test the cell membrane expression of CD55 and CD59 following PI-PLC exposure, SK-BR-3 and BT474 cells were treated with $0.1 \mathrm{U} / \mathrm{ml}$ PI-PLC for $1 \mathrm{~h}$ at $37^{\circ} \mathrm{C}$ prior to staining and flow cytometry.

Immunocytochemical staining for HER2. Cells were seeded in 6 -well plates at a concentration of $5 \times 10^{5}$ cells/well for $24 \mathrm{~h}$, then fixed with cold methanol for $15 \mathrm{~min}$. Cells were incubated with primary antibodies against HER2 (dilution, 1:200 in PBS) overnight at $4^{\circ} \mathrm{C}$. Cells were incubated with the appropriate secondary antibodies for $60 \mathrm{~min}$ at $37^{\circ} \mathrm{C}$. A DAB color developing system, and hematoxylin and eosin staining were used for the following steps. The negative controls were created by replacing the primary antibodies with PBS. Stained cells were observed by light microscope and 5 fields of view were counted by eye for cell numbers according to the following scoring system: $0=$ negative, no dye or $<10 \%$ cells with cell membrane staining; $1+=$ weak positive, $>10 \%$ cells with thin, fragmented cell membrane staining; $2+=$ positive, $>10 \%$ cells with thin to moderate intact cell membrane staining; and $3+=$ strong positive/high expression, $>30 \%$ cells with moderate to thick intact cell membrane staining.

Trypan blue exclusion assay. SK-BR-3, BT474 and PIEC cells were removed from culture bottle using $0.25 \%$ trypsin and $0.25 \%$ EDTA, Single cells were suspended in PBS, counted and divided into $10^{6}$ cells per Eppendorf tube. SK-BR-3 and BT474 cells in each Eppendorf tube were centrifuged at $500 \mathrm{x} \mathrm{g}$ and incubated with $50 \%$ INHS, $50 \% \mathrm{NHS}, 50 \mu \mathrm{g} / \mathrm{ml}$ trastuzumab or $50 \mu \mathrm{g} / \mathrm{ml}$ trastuzumab $+50 \%$ NHS in a total volume of $500 \mu \mathrm{l}$ for $1 \mathrm{~h}$ at $37^{\circ} \mathrm{C}$. PIEC cells were incubated with $50 \%$ INHS or $50 \%$ NHS. A total of $100 \mu \mathrm{l}$ of cell suspension was added into an equal volume of $0.4 \%$ trypan blue, then the number of living/dead cells were counted, and the survival and lysis rates were calculated as follows: Survival rate $(\%)=$ Number of living cells/(number of living cells + number of dead cells) $x 100$; lysis rate $=100 \%$ - survival rate.

In order to block CD55 and CD59, SK-BR-3 and BT474 cells were pre-incubated with $50 \mu \mathrm{g} / \mathrm{ml}$ trastuzumab and $10 \mu \mathrm{g} / \mathrm{ml}$ anti-CD55 or anti-CD59 $\mathrm{mAbs}$ for $10 \mathrm{~min}$ at room temperature, then $50 \%$ NHS was added to make up a final volume of $500 \mu \mathrm{l}$. The samples were then incubated for $1 \mathrm{~h}$ at $37^{\circ} \mathrm{C}$.

For the PI-PLC pre-treatment, SK-BR-3 and BT474 cells were incubated with $0.01,0.05,0.1$ and $0.2 \mathrm{U} / \mathrm{ml}$ (diluted in PBS) for $1 \mathrm{~h}$ at $37^{\circ} \mathrm{C}$. Cells were then incubated with $50 \mu \mathrm{g} / \mathrm{ml}$ trastuzumab $+50 \%$ NHS (final volume, $500 \mu \mathrm{l}$ ). Each experiment was repeated three times.

Downregulation of CD55 and CD59 expression with shRNA in $S K-B R-3$ cells. The shRNA plasmids $(4 \mu \mathrm{g})$ were mixed with $10 \mu \mathrm{l}$ Lipofectamine 2000 in $500 \mu \mathrm{l}$ serum-free DMEM and transfected into SK-BR-3 cells to knockdown the expression of
CD55 and CD59. Following transfection with shRNA for $48 \mathrm{~h}$, the interference efficiencies of shRNAs were determined by RT-qPCR and western blot analysis. A trypan blue exclusion assay was also tested according to the aforementioned method.

RNA extraction and RT-qPCR analysis. Total RNA was isolated from SK-BR-3 cells using RNeasy Mini kit (cat no. 74104; Qiagen GmbH, Hilden, Germany) according to the manufacturer's protocol. First-strand cDNAs were synthesized from total RNA using 5X All-In-One RT MasterMix (G492; Applied Biological Materials, Inc., Richmond, BC, Canada) according to the manufacturer's protocol. RT-qPCR was performed using SsoFast ${ }^{\mathrm{TM}}$ Eva Green ${ }^{\circledR}$ Supermix (cat no. 172) in a Chromo4 Real-Time PCR detector (both from Bio-Rad Laboratories, Inc.), according to the manufacturer's protocol. Cycling conditions for the RT-qPCR were $95^{\circ} \mathrm{C}$ for $30 \mathrm{sec}$, followed by 40 cycles of $95^{\circ} \mathrm{C}$, for $5 \mathrm{sec}$ and $60^{\circ} \mathrm{C}$ for $10 \mathrm{sec}$. The primer sequences used were described in previous sections. The relative quantitation of gene expression was performed using the $2^{-\Delta \Delta \mathrm{Cq}}$ method (12) with GAPDH as a reference gene. Experiments were repeated three times.

Western blot analysis. Whole cells were lysed at $4{ }^{\circ} \mathrm{C}$ in radioimmunoprecipitation lysis buffer (Beyotime Institute of Biotechnology, Shanghai, China). Total protein concentration was determined using the BCA kit (Beyotime Institute of Biotechnology) according to the manufacturer's protocol. A total of $35 \mu \mathrm{g}$ protein from each group was separated by $12 \%$ SDS-PAGE and then transferred to polyvinylidene fluoride membranes. The membranes were blocked with $5 \%$ nonfat milk in PBS-Tween ( $0.1 \%$ Tween in PBS). The membranes were incubated at $4^{\circ} \mathrm{C}$ overnight with the primary antibodies against CD55 (dilution, 1:400), CD59 (dilution, 1:1,000) and $\beta$-actin (dilution, 1:1,000). Subsequent to washing with PBS-Tween three times for $10 \mathrm{~min}$, the membranes were incubated at room temperature for $2 \mathrm{~h}$ with HRP-labeled goat anti-mouse IgG (dilution, 1:2,000) and goat anti-rat IgG (dilution, 1:10,000) secondary antibodies. Subsequent to washing with PBS-Tween 3 times for $10 \mathrm{~min}$, the bands were visualized using chemiluminescent HRP substrate (cat no. WBKLS0100) (EMD Millipore), and detected using the ChemiDoc XRS kit (Bio-Rad, Laboratories, Inc.) according to the manufacturer's protocol.

Statistical analysis. The data were expressed as the mean \pm standard deviation. Statistical analysis was performed as analysis of variance followed by one-way analysis of variance for experiments consisting of more than two groups. A t-test was used for comparison between two groups. $\mathrm{P}<0.05$ was considered to indicate a statistically significant difference. SPSS software version 16.0 (SPSS Inc., Chicago, IL, USA) was used.

\section{Results}

Expression of CD55 and CD59 in breast cancer cells. The surface expression of CD55 and CD59 in breast cancer cells MCF-7, SK-BR-3, MDA-MB-468, BT549, MDA-MB-231, BT474 and ZR-75-1, and the human colorectal adenocarcinoma cell line LoVo was evaluated by FCM (Table I). All 
Table I. MFI of CD55 and CD59 in 7 breast cancer cell lines as detected by flow cytometry.

\begin{tabular}{lcc}
\hline Cell line & CD55 ${ }^{\text {a }}$ MFI & CD59a, MFI \\
\hline SK-BR-3 & $741 \pm 92$ & $5257 \pm 283$ \\
MCF-7 & $565 \pm 55$ & $4379 \pm 135$ \\
ZR-75-1 & $324 \pm 99$ & $2736 \pm 265$ \\
MDA-MB-468 & $393 \pm 36$ & $9172 \pm 171$ \\
BT549 & $291 \pm 57$ & $4710 \pm 157$ \\
MDA-MB-231 & $2242 \pm 153$ & $4402 \pm 153$ \\
BT474 & $844 \pm 98$ & $7965 \pm 342$ \\
LoVo & $35 \pm 21$ & $119 \pm 17$ \\
\hline
\end{tabular}

${ }^{\text {a}}$ Data are expressed as the mean \pm standard deviation. MFI, mean fluorescence intensity.

the breast cancer cell lines evaluated exhibited markedly increased CD55 and CD59 expression compared with LoVo cells [negative control (9)].

Expression of HER2 in breast cancer cells. Immunocytochemical staining was performed to determine the expression level of HER 2 protein in the 7 breast cancer cell lines. The results demonstrated HER2 strong positive (3+) expression in SK-BR-3, BT474 and ZR-75-1 cells, and negative (0) expression in other cell lines (Fig. 1). SK-BR-3 and BT474 cells were selected for subsequent experiments as isolated single ZR-75-1 cells were difficult to obtain via trypsin digestion, and the resultant cell clusters may cause inaccuracies in subsequent trypan blue cell counting experiments.

Trastuzumab does not induce CDC in SK-BR-3 and BT474 cells. Trypan blue exclusion assays demonstrated that NHS treatment significantly increased PIEC cell death compared with INHS treatment $(\mathrm{P}<0.05$; Fig. 2). This confirmed that the NHS was suitable for subsequent experiments. No significant differences were observed between the cell lysis rates for SK-BR-3 and BT474 cells treated with INHS, NHS, trastuzumab and trastuzumab + NHS (P>0.05; Fig. 2). Cell survival rates were $>97 \%$ in these treatment groups. The data indicated that trastuzumab does not induce CDC in SK-BR-3 and BT474 cells.

Co-treatmentwith trastuzumaband anti-CD55/59mAbs results in $C D C$-dependent cell lysis. Trypan blue exclusion assays demonstrated that, in the trastuzumab + anti-CD55, trastuzumab + anti-CD59, trastuzumab + anti-CD55 + anti-CD59 treatment groups, the cell lysis rates following NHS treatment for SK-BR-3 were 14.3, 24.2 and 39.5\%, respectively (Fig. 3A), and for BT474 were $18.69,24.95$ and $32.37 \%$, respectively (Fig. 3B). The cell lysis rates in these groups were significantly increased compared with the trastuzumab and anti-CD55/59 alone treatment groups $(\mathrm{P}<0.05)$. In addition, the cell lysis rate for the trastuzumab + anti-CD55 + anti-CD59 treatment group was significantly increased compared with the trastuzumab + anti-CD55 or the trastuzumab + anti-CD59 treatment groups $(\mathrm{P}<0.05)$. These results indicate that
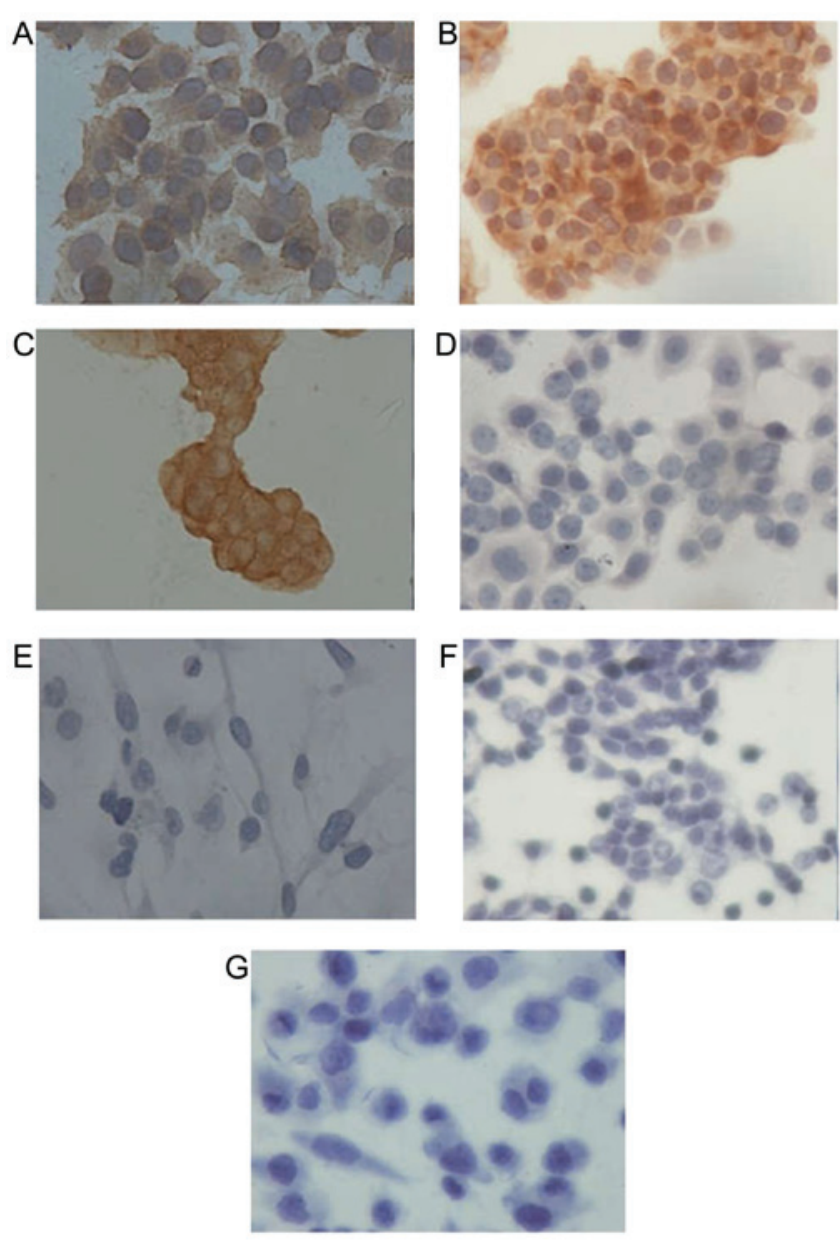

Figure 1. Human epidermal growth factor receptor 2 expression in 7 breast cancer cell lines as detected by immunohistochemical staining. (A) SK-BR-3, (B) BT474, (C) ZR-75-1, (D) MDA-MB-468, (E) MDA-MB-231, (F) MCF-7 and (G) BT549 cells. Magnification, $\mathrm{x} 400$.

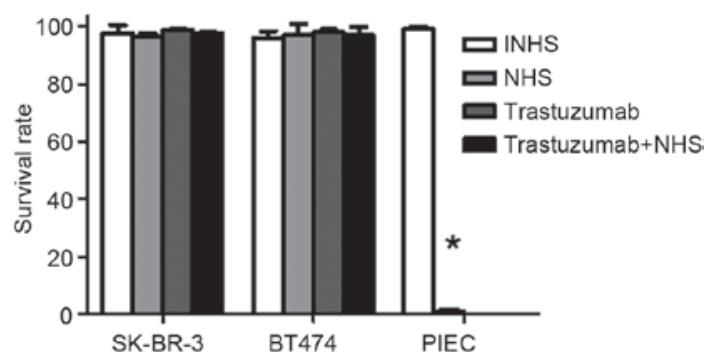

Figure 2. Cell survival rates of SK-BR-3, BT474 and PIEC cells following treatment with INHS, NHS and/or trastuzumab. SK-BR-3, BT474 cells incubated with 50\% INHS, $50 \%$ NHS, $50 \mu \mathrm{g} / \mathrm{ml}$ trastuzumab and $50 \mu \mathrm{g} / \mathrm{ml}$ trastuzumab $+50 \% \mathrm{NHS}$ for $1 \mathrm{~h}$ at $37^{\circ} \mathrm{C}$. PIEC cells were included as a positive control ( $100 \%$ lysis rate in NHS). ${ }^{*} \mathrm{P}<0.05$ compared with INHS group. INHS, inactivated normal human serum; NHS, normal human serum.

co-treatment of cells with trastuzumab and anti-CD55/59 mAbs results in CDC-dependent lysis of SK-BR-3 and BT474 cells.

PI-PLC promotes trastuzumab-induced CDC in SK-BR-3 and BT474 cells in a dose-dependent manner through cleavage of CD55 and CD59. A trypan blue exclusion assay demonstrated 

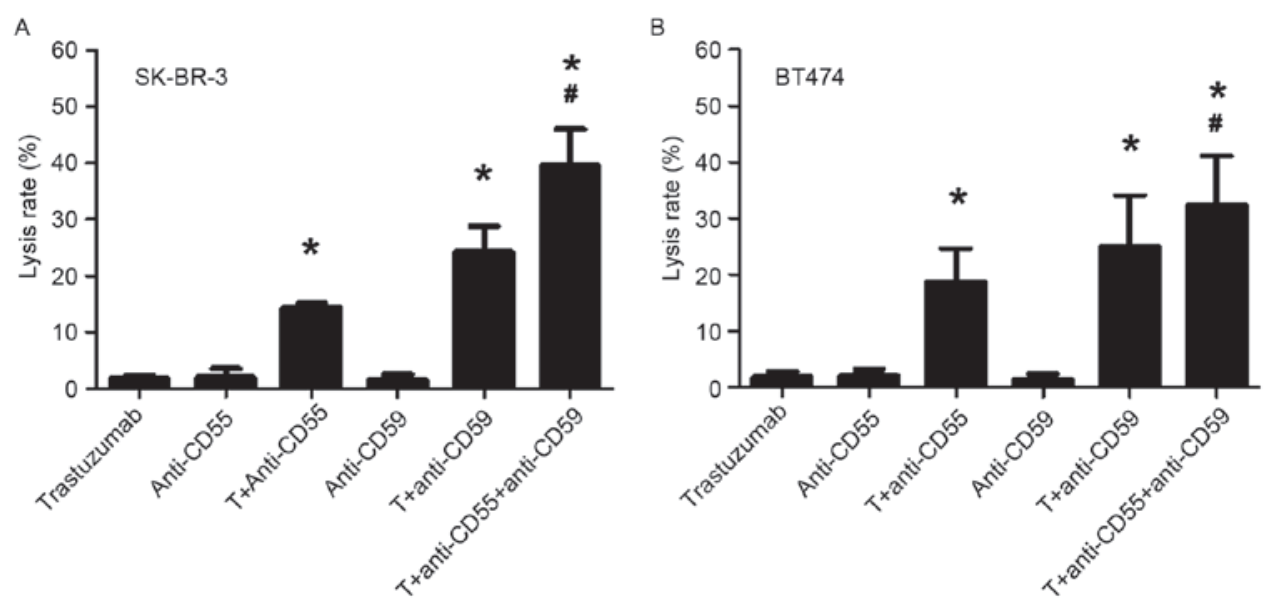

Figure 3. Effect of anti-CD55 and anti-CD59 on trastuzumab-induced CDC in SK-BR-3 and BT474 cells. Lysis rates of (A) SK-BR-3 and (B) BT474 cells following pre-incubation with $50 \mu \mathrm{g} / \mathrm{ml}$ trastuzumab and each $\mathrm{mAb}$ (anti-CD55, anti-CD59, anti-CD55 + anti-CD59) (10 $\mu \mathrm{g} / \mathrm{ml})$ for $10 \mathrm{~min}$ at room temperature, then subsequent addition of 50\% NHS and incubation for $1 \mathrm{~h}$ at $37^{\circ} \mathrm{C} .{ }^{*} \mathrm{P}<0.05$ compared with trastuzumab, anti-CD55 and anti-CD59 alone groups ${ }^{\text {"P}} \mathrm{P}<0.05$ compared with the $\mathrm{T}+$ anti-CD55 and $\mathrm{T}+$ anti-CD59 groups. T, trastuzumab; CDC, complement-dependent cytotoxicity; CD55, complement decay-accelerating factor; CD59, CD59 glycoprotein precursor.

that following treatment with NHS, trastuzumab-induced CDC was significantly enhanced in PI-PLC pre-treated SK-BR-3 and BT474 cells in a dose-dependent manner ( $\mathrm{P}<0.05$; Fig. 4A). The cell lysis rate reached a maximum with pre-treatment with $0.1 \mathrm{U} / \mathrm{ml}$ PI-PLC (33.5 and $37.7 \%$ for SK-BR-3 and BT474 cells, respectively). Following treatment with $0.1 \mathrm{U} / \mathrm{ml}$ PI-PLC for $1 \mathrm{~h}$, FCM analysis revealed a significant decrease in CD55/59 fluorescence signal compared with before PI-PLC treatment $(\mathrm{P}<0.05$; Fig. $4 \mathrm{~B}$ and $\mathrm{C})$, indicating that $\mathrm{CD} 55$ and CD59 had been cleaved.

Confirmation of CD55 and CD59 downregulation following shRNA transfection of $S K-B R-3$ cells. RT-qPCR analysis demonstrated that CD59 mRNA expression was significantly downregulated following shCD59 transfection compared with the shNC and control groups $(\mathrm{P}<0.05$; Fig. 5A). The 4 shRNA fragments targeting CD55 exhibited different efficiencies in downregulating CD55 mRNA. Treatment with shCD55/545 or shCD55/829 demonstrated the highest downregulation efficiencies, by 84.5 and $82 \%$, respectively, compared with the shNC control $(\mathrm{P}<0.05$; Fig. $5 \mathrm{~B})$.

Western blot analysis demonstrated that CD59 protein expression was downregulated following treatment with shCD59 compared with the shNC and control groups (Fig. 5C). In addition, CD55 protein expression was downregulated following treatment with shCD55/545 or shCD55/829 comparing with shNC and control groups (Fig. 5D). The shCD55/545 fragment demonstrated markedly increased downregulation efficiency compared with shCD55/829; therefore shCD55/545-transfected SK-BR-3 cells were selected for subsequent experiments.

A Trypan blue exclusion assay revealed that transfection with shCD59 resulted in a significantly increased lysis rate following trastuzumab treatment compared with control shNC cells (17.3 vs. $2.3 \%$; $\mathrm{P}<0.05$; Fig. $6 \mathrm{~A})$. In addition, transfection with shCD55/545 resulted in a significantly increased lysis rate following trastuzumab treatment compared with control shNC cells (24.3 vs. $1.9 \%$; $\mathrm{P}<0.05$; Fig. 6B).

\section{Discussion}

A number of mAbs have been authorized for the treatment of patients. The molecular mechanisms underlying the anti-tumor effects of mAbs include the inhibition of signaling pathways, which lead to downstream effects, including apoptosis, blocking of growth factor receptor and inhibition of angiogenesis $(4,13)$. Although a number of studies have demonstrated that mAbs exhibit low efficacy against their targets (14), mAbs may exhibit desirable effects beyond target-related pathways, including enhancement of CDC.

Trastuzumab comprises a human immunoglobulin G1 Fc segment, a potent enhancer of CDC $(15,16)$. However, previous studies have indicated that effects on CDC effects are not a key molecular mechanism underlying the anti-tumor effects of trastuzumab (5,6,17-19). Abnormal expression of mCRPs has been reported in a number of types of tumor (7,20-26), which may inhibit the activation of complement factors and $\mathrm{CDC}$ effects induced by $\mathrm{mAbs}$, therefore compromising the therapeutic potential of mAbs (23). In accordance with previous studies, which reported that breast cancer cells overexpress mCRPs $(25,27-29)$, all 7 breast cancer cell lines used in the present study were demonstrated to overexpress CD55 and CD59. The HER2-positive cell lines SK-BR-3 and BT474 were selected for subsequent study. Treatment with trastuzumab was not sufficient to induce CDC in these cells in the presence of NHS, which is consistent with the results from Mineo et al (5), which demonstrated that trastuzumab could not induce CDC in CD55/59-expressing malignant neuroblastoma cells. Therefore, the failure of trastuzumab to induce CDC in HER-2-positive breast cancer cells may be due to the high expression of CD55 and CD59.

Previous reports have indicated that blocking the biological function of mCRPs may enhance the anti-tumor effects of mAbs. For example, rituximab is a human-mouse chimeric antibody that targets malignant non-Hodgkin's lymphoma B cells that overexpress CD20. The expression of CD55 and CD59 on the surface of lymphoma cells was associated with 

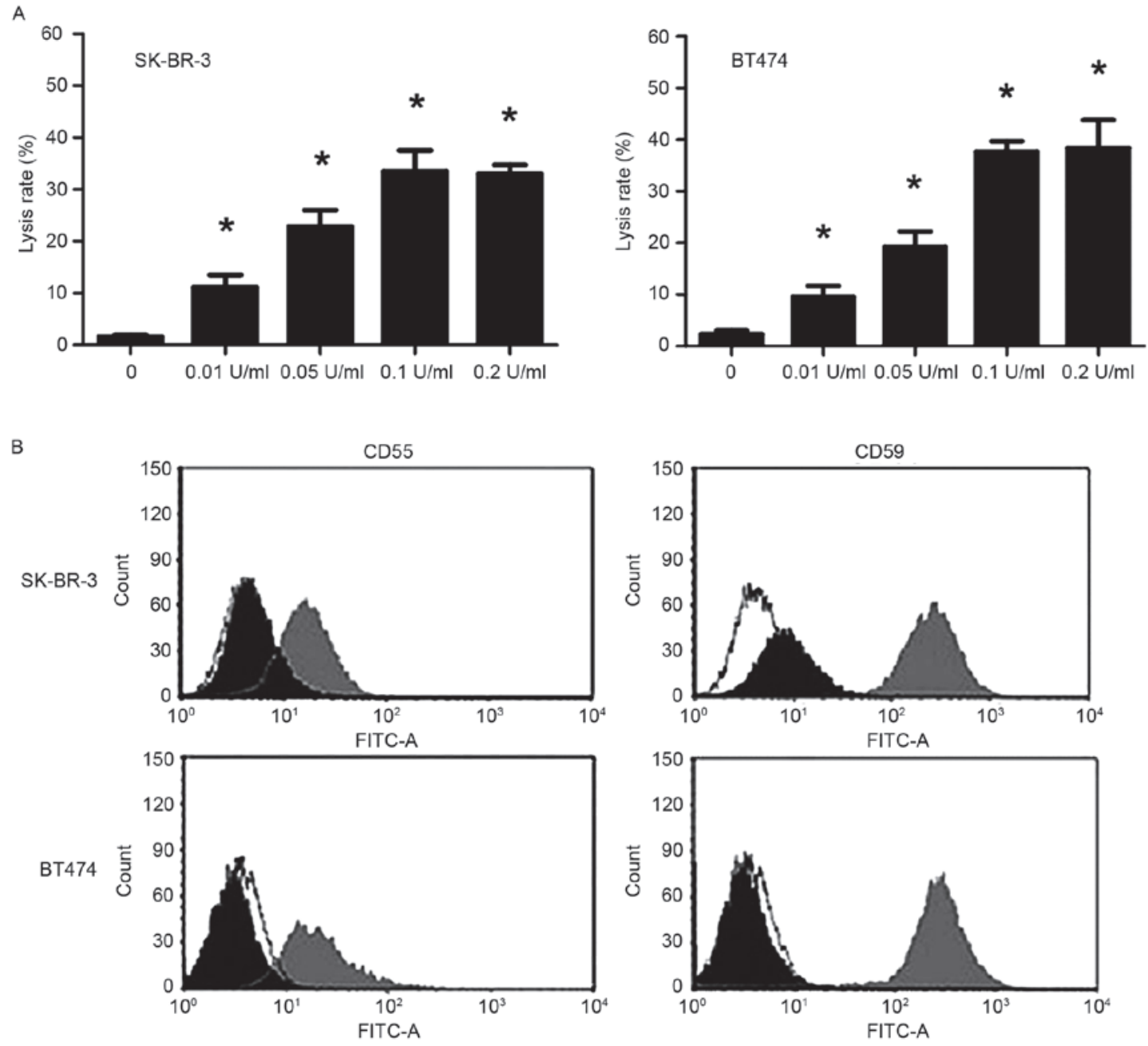

C
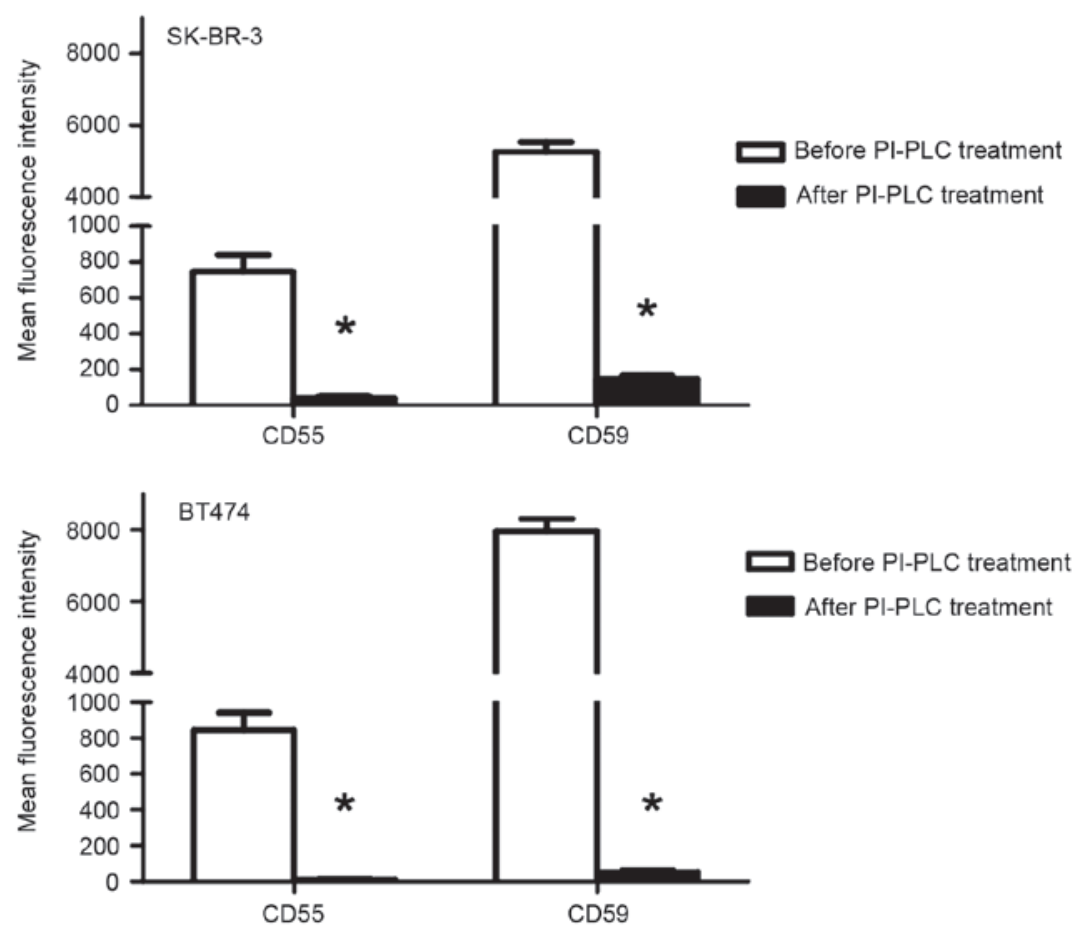

Figure 4. Effect of PI-PLC pre-treatment on trastuzumab-induced CDC in SK-BR-3 and BT474 cells. (A) Lysis rates in SK-BR-3 and BT474 cells following pre-treatment with PI-PLC $(0.01,0.05,0.1$ and $0.2 \mathrm{U} / \mathrm{ml})$ cells for $1 \mathrm{~h}$ at $37^{\circ} \mathrm{C}$ followed by incubation with $50 \mu \mathrm{g} / \mathrm{ml}$ trastuzumab $+50 \% \mathrm{NHS}$. ${ }^{*} \mathrm{P}<0.05$, compared with the control $(0 \mathrm{U} / \mathrm{ml})$. (B) Representative flow cytometry histograms (white, control of similar type; gray, pre-treatment; black, post-treatment) and (C) quantification of CD55 and CD59 expression in SK-BR-3 and BT474 cells following $0.1 \mathrm{U} / \mathrm{ml}$ PI-PLC treatment. "P<0.05, compared with before PI-PLC treatment. CDC, complement-dependent cytotoxicity; CD55, complement decay-accelerating factor; CD59, CD59 glycoprotein precursor; PI-PLC, phosphatidylinositol-specific phospholipase C. 


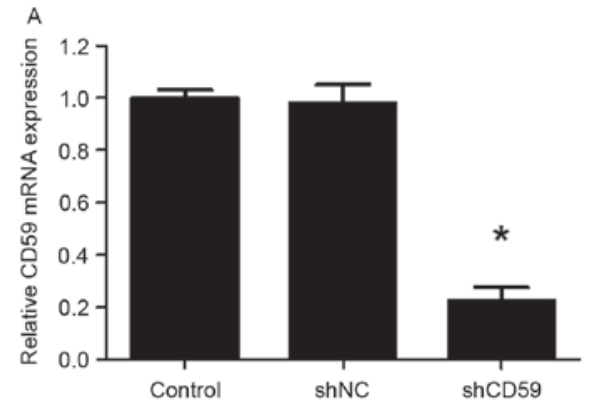

C

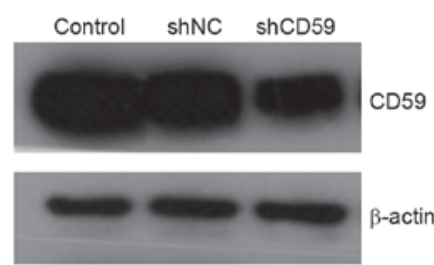

B

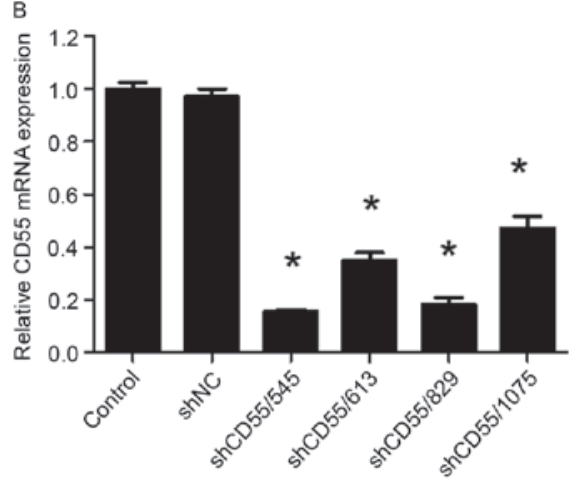

D

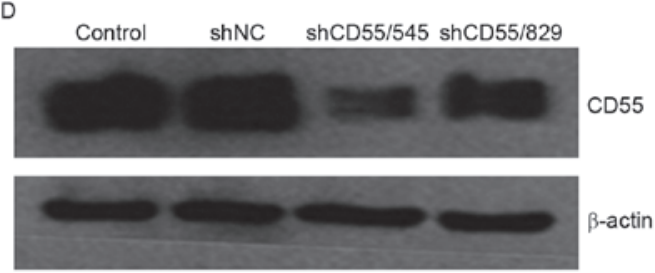

Figure 5. Downregulation of CD59 and CD55 in SK-BR-3 cells following treatment with shRNA. RT-qPCR analysis of (A) CD59 and (B) CD55 expression and western blot analysis of (C) CD59 and (D) CD55 expression following transfection with shNC, shCD59 or 4 specific shRNA fragments for CD55 for $48 \mathrm{~h}$. SK-BR-3 cells without transfection were used as a control. GAPDH served as an internal control for RT-qPCR. $\beta$-actin protein levels served as the loading control for western blot analysis. " $\mathrm{P}<0.05$, compared with the control and shNC groups. CDC, complement-dependent cytotoxicity; CD55, complement decay-accelerating factor; CD59, CD59 glycoprotein precursor; NC, negative control; shRNA, short hairpin RNA; RT-qPCR, reverse transcription-quantitative polymerase chain reaction.
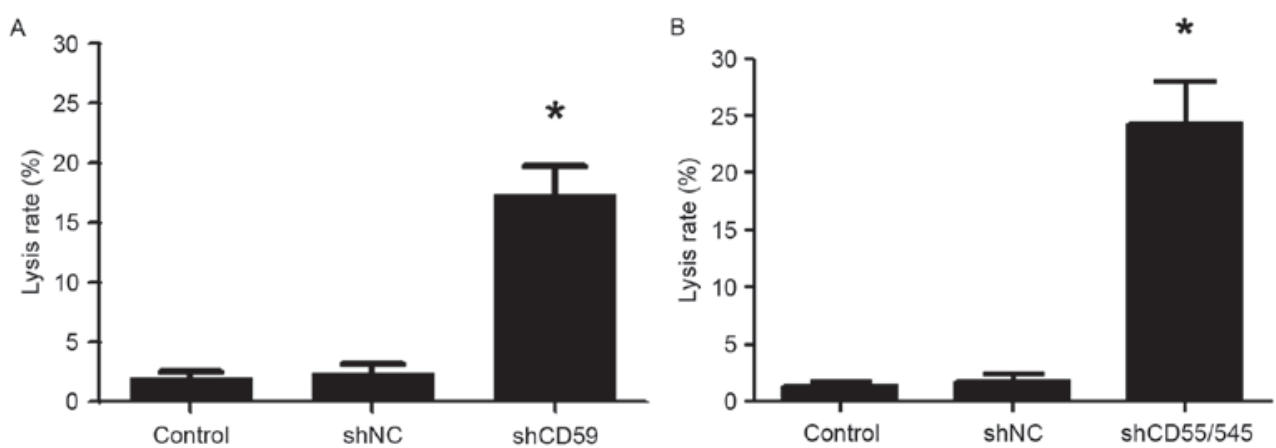

Figure 6. Effect of specific downregulation of CD55 and CD59 on trastuzumab-induced CDC of SK-BR-3 cells. Lysis rates of SK-BR-3 cells following transfection with shNC, and (A) shCD59 or (B) shCD55/545 for $48 \mathrm{~h}$, followed by incubation with $50 \mu \mathrm{g} / \mathrm{ml}$ trastuzumab+50\% NHS. Non-transfected SK-BR-3 cells were included as a control. ${ }^{*} \mathrm{P}<0.05$ compared with the control and shNC groups. CDC, complement-dependent cytotoxicity; CD55, complement decay-accelerating factor; CD59, CD59 glycoprotein precursor; NC, negative control; shRNA, short hairpin RNA.

their resistance to CDC. Lymphoma cells that highly expressed CD55 and CD59 exhibited a lower cytolysis rate induced by rituximab (30-32). These previous studies have revealed that blocking, degradation and downregulation of mCRPs significantly promote $\mathrm{CDC}$ effects induced by rituximab in lymphoma cells.

In the present study, blocking antibodies targeting CD55 or CD59 significantly enhanced trastuzumab-induced CDC. The cytolysis rate increased from $1-2 \%$ up to $14-25 \%$ and the combined use of the two antibodies further increased the cytolysis rate to $32-40 \%$. The primary biological function of CD55 is to prevent the assembly of C3 and C5 transferase and accelerate their decay. The primary biological function of CD59 is preventing the formation of the membrane attack complex by binding to $\mathrm{C} 8$ and $\mathrm{C} 9$ in the final stage of the complement activation phase $(33,34)$.
PI-PLC can specifically separate glycosyl-phosphatidyl inositol-anchored CD55 and CD59 from the cell membrane. Previous research demonstrated that the sensitivity of melanoma (35), lung carcinoma (36) and ovarian cells (37) to CDC was promoted by PI-PLC. Results from the present study demonstrated that PI-PLC enhances the sensitivity of SK-BR-3 and BT474 cells to trastuzumab-induced CDC. PI-PLC pre-treatment increased the cell lysis rate to $30-40 \%$, which was comparable with the lysis rate following combined treatment with CD55 and CD59 blocking antibodies. The effects of PI-PLC on the cell lysis rate increased in a concentration-dependent manner up to $0.1 \mathrm{U} / \mathrm{ml}$.

Small interfering RNA (siRNA)-mediated RNA interference is currently the most effective method for specific gene silencing (38). Bellone et al (39) indicated that downregulation of CD55 and CD59 by siRNA enhances trastuzumab-induced 
CDC in uterine serous carcinoma cells, but the knockdown of CD55 and CD59 were only 11.6 and $10.7 \%$, respectively.

Mamidi et al (40) reported that knockdown of mCRPs (CD46, CD55 and CD59) by chemically stabilized siRNAs using cationic lipoplexes (AtuPLEXes) led to increased CDC in BT474, SK-BR-3 (breast), SKOV3 (ovarian) and Calu-3 (lung) cancer cell lines following combined treatment with trastuzumab and pertuzumab. However, knockdown of individual mCRPs did not significantly increase trastuzumab-induced CDC. Application of in vivo siRNA technologies is a rapid development field with many challenges, including the development of transfection systems, instability and adverse responses in vivo. shRNA plasmids can integrate into the host genome and achieve long-term gene silencing. In the present study, shRNA plasmids were designed that targeted CD55 and CD59 separately. The shRNAs downregulated CD55 and CD59 at mRNA and protein levels, and significantly increased the cytolytic effect of trastuzumab-induced CDC. Downregulation of CD55 or CD59 resulted in a cytolysis rate of 24.3 and $17.3 \%$, respectively. Therefore, downregulation of CD55 and CD59 can enhance trastuzumab-induced CDC.

The results from the present study indicate that PI-PLC could almost completely split CD55 and CD59 from cell membrane specifically, but the cytolysis rate only reached a maximum of $\sim 40 \%$. Thus, other mCRPs may serve crucial regulatory roles. Future studies are required to investigate the role of other complement regulatory proteins, including soluble and membrane-bound components.

Together with previous research (10), the results from the present study indicate that mCRP expression may be a predictor of patient prognosis and response to trastuzumab. The combined application of mAbs, RNA interference, or other means of downregulating $\mathrm{mCRP}$ expression may improve the clinical efficacy of trastuzumab.

\section{Acknowledgements}

The present study was supported by the National Natural Science Foundation of China (grant no. 30972940).

\section{References}

1. Servick K: Breast cancer. Breast cancer: A world of differences. Science 343: 1452-1453, 2014.

2. Thomas E and Berner G: Prognostic and predictive implications of HER2 status for breast cancer patients. Eur J Oncol Nurs 4: 10-17, 2000.

3. Cardoso F, Piccart MJ, Durbecq V and Di Leo A: Resistance to trastuzumab: A necessary evil or a temporary challenge? Clin Breast Cancer 3: 247-259, 2002.

4. Scott AM, Wolchok JD and Old LJ: Antibody therapy of cancer. Nat Rev Cancer 12: 278-287, 2012.

5. Mineo JF, Bordron A, Quintin-Roué I, Loisel S, Ster KL, Buhé V, Lagarde N and Berthou C: Recombinant humanised anti-HER2/neu antibody (Herceptin) induces cellular death of glioblastomas. Br J Cancer 91: 1195-1199, 2004

6. Prang N, Preithner S, Brischwein K, Göster P, Wöppel A, Müller J, Steiger C, Peters M, Baeuerle PA and da Silva AJ: Cellular and complement-dependent cytotoxicity of Ep-CAM-specific monoclonal antibody MT201 against breast cancer cell lines. Br J Cancer 92: 342-349, 2005.

7. Fishelson Z, Donin N, Zell S, Schultz S and Kirschfink M: Obstacles to cancer immunotherapy: Expression of membrane complement regulatory proteins (mCRPs) in tumors. Mol Immunol 40: 109-123, 2003.
8. Macor P and Tedesco F: Complement as effector system in cancer immunotherapy. Immunol Lett 111: 6-13, 2007.

9. Wu Y, Wang Y, Qin F, Wang Z, Wang Y, Yang Y, Zheng H and Zheng H: CD55 limits sensitivity to complement-dependent cytolysis triggered by heterologous expression of $\alpha$-gal xenoantigen in colon tumor cells. Am J Physiol Gastrointest Liver Physiol 306: G1056-G1064, 2014.

10. Liu M, Yang YJ, Zheng H, Zhong XR, Wang Y, Wang Z, Wang YG and Wang YP: Membrane-bound complement regulatory proteins are prognostic factors of operable breast cancer treated with adjuvant trastuzumab: A retrospective study. Oncol Rep 32: 2619-2627, 2014.

11. Shi XX, Zhang B, Zang JL, Wang GY and Gao MH: CD59 silencing via retrovirus-mediated RNA interference enhanced complement-mediated cell damage in ovary cancer. Cell Mol Immunol 6: 61-66, 2009.

12. Livak KJ and Schmittgen TD: Analysis of relative gene expression data using real-time quantitative PCR and the 2(-Delta Delta C(T)) method. Methods 25: 402-408, 2001.

13. Reichert JM: Antibody-based therapeutics to watch in 2011. MAbs 3: 76-99, 2011.

14. Strome SE, Sausville EA and Mann D: A mechanistic perspective of monoclonal antibodies in cancer therapy beyond target-related effects. Oncologist 12: 1084-1095, 2007.

15. Clark MR: IgG effector mechanisms. Chem Immunol 65: 88-110, 1997.

16. Dalle S, Thieblemont C, Thomas L and Dumontet C: Monoclonal antibodies in clinical oncology. Anticancer Agents Med Chem 8: 523-532, 2008

17. Dean-Colomb W and Esteva FJ: Her2-positive breast cancer: Herceptin and beyond. Eur J Cancer 44: 2806-2812, 2008.

18. De Lorenzo C, Tedesco A, Terrazzano G, Cozzolino R, Laccetti P, Piccoli R and D'Alessio G: A human, compact, fully functional anti-ErbB2 antibody as a novel antitumour agent. Br J Cancer 91: 1200-1204, 2004.

19. Yu J, Caragine T, Chen S, Morgan BP, Frey AB and Tomlinson S: Protection of human breast cancer cells from complement-mediated lysis by expression of heterologous CD59. Clin Exp Immunol 115: 13-18, 1999.

20. Gelderman KA, Tomlinson S, Ross GD and Gorter A: Complement function in $\mathrm{mAb}$-mediated cancer immunotherapy. Trends Immunol 25: 158-164, 2004.

21. Yan J, Allendorf DJ, Li B, Yan R, Hansen R and Donev R: The role of membrane complement regulatory proteins in cancer immunotherapy. Adv Exp Med Biol 632: 159-174, 2008.

22. Jurianz K, Ziegler S, Garcia-Schüler H, Kraus S, Bohana-Kashtan O, Fishelson Z and Kirschfink M: Complement resistance of tumor cells: Basal and induced mechanisms. Mol Immunol 36: 929-939, 1999.

23. Gancz D and Fishelson Z: Cancer resistance to complement-dependent cytotoxicity (CDC): Problem-oriented research and development. Mol Immunol 46: 2794-2800, 2009.

24. Rushmere NK, Knowlden JM, Gee JM, Harper ME, Robertson JF, Morgan BP and Nicholson RI: Analysis of the level of mRNA expression of the membrane regulators of complement, CD59, CD55, and CD46, in breast cancer. Int J Cancer 108: 930-936, 2004.

25. Ravindranath NM and Shuler C: Cell-surface density of complement restriction factors (CD46, CD55 and CD59): Oral squamous cell carcinoma versus other solid tumors. Oral Surg Oral Med Oral Pathol Oral Radiol Endod 103: 231-239, 2007.

26. Loberg RD, Wojno KJ, Day LL and Pienta KJ: Analysis of membrane-bound complement regulatory proteins in prostate cancer. Urology 66: 1321-1326, 2005.

27. Madjd Z, Durrant LG, Bradley R, Spendlove I, Ellis IO and Pinder SE: Loss of CD55 is associated with aggressive breast tumors. Clin Cancer Res 10: 2797-2803, 2004.

28. Zell S, Geis N, Rutz R, Schultz S, Giese T and Kirschfink M: Down-regulation of CD55 and CD46 expression by anti-sense phosphorothioate oligonucleotides (S-ODNs) sensitizes tumour cells to complement attack. Clin Exp Immunol 150: 576-584, 2007.

29. Jurianz K, Maslak S, Garcia-Schuler H, Fishelson Z and Kirschfink M: Neutralization of complement regulatory proteins augments lysis of breast carcinoma cells targeted with rhum $\mathrm{Ab}$ anti-HER2. Immunopharmacology 42: 209-218, 1999.

30. Macor P, Tripodo C, Zorzet S, Piovan E, Bossi F, Marzari R, Amadori A and Tedesco F: In vivo targeting of human neutralizing antibodies against CD55 and CD59 to lymphoma cells increases the antitumor activity of rituximab. Cancer Res 67: 10556-10563, 2007. 
31. Golay J, Lazzari M, Facchinetti V, Bernasconi S, Borleri G, Barbui T, Rambaldi A and Introna M: CD20 levels determine the in vitro susceptibility to rituximab and complement of B-cell chronic lymphocytic leukemia: Further regulation by CD55 and CD59. Blood 98: 3383-3389, 2001.

32. Zhou X, Hu W and Qin X: The role of complement in the mechanism of action of rituximab for B-cell lymphoma: Implications for therapy. Oncologist 13: 954-966, 2008.

33. Nicholson-Weller A and Wang CE: Structure and function of decay accelerating factor CD55. J Lab Clin Med 123: 485-491, 1994.

34. Fonsatti E, Altomonte M, Coral S, De Nardo C, Lamaj E, Sigalotti L, Natali PG and Maio M: Emerging role of protectin (CD59) in humoral immunotherapy of solid malignancies. Clin Ter 151: 187-193, 2000

35. Brasoveanu LI, Altomonte M, Fonsatti E, Colizzi F, Coral S, Nicotra MR, Cattarossi I, Cattelan A, Natali PG and Maio M: Levels of cell membrane CD59 regulate the extent of complement-mediated lysis of human melanoma cells. Lab Invest 74: 33-42, 1996

36. Azuma A, Yamano Y, Yoshimura A, Hibino T, Nishida T, Yagita H, Okumura K, Seya T, Kannagi R, Shibuya M, et al: Augmented lung adenocarcinoma cytotoxicity by the combination of a genetically modified anti-Lewis Y antibody and antibodies to complement regulatory proteins. Scand J Immunol 42: 202-208, 1995.
37. Bjorge L, Hakulinen J, Wahlström T, Matre R and Meri S: Complement-regulatory proteins in ovarian malignancies. Int $\mathrm{J}$ Cancer 70: 14-25, 1997.

38. Aagaard L and Rossi JJ: RNAi therapeutics: Principles, prospects and challenges. Adv Drug Deliv Rev 59: 75-86, 2007.

39. Bellone S, Roque D, Cocco E, Gasparrini S, Bortolomai I, Buza N, Abu-Khalaf M, Silasi DA, Ratner E, Azodi M, et al: Downregulation of membrane complement inhibitors CD55 and CD59 by siRNA sensitises uterine serous carcinoma overexpressing Her2/neu to complement and antibody-dependent cell cytotoxicity in vitro: Implications for trastuzumab-based immunotherapy. Br J Cancer 106: 1543-1550, 2012.

40. Mamidi S, Cinci M, Hasmann M, Fehring V and Kirschfink M: Lipoplex mediated silencing of membrane regulators (CD46, CD55 and CD59) enhances complement-dependent anti-tumor activity of trastuzumab and pertuzumab. Mol Oncol 7: 580-594, 2013. 